\title{
CONCEPTO HOSTOSIANO DEL SER HUMANO: ESBOZO PARA UN MODELO PUERTORRIQUEÑO DE TRABAJO SOCIAL
}




\section{lleana Carrión Maldonado}

Posee Maestría en Trabajo Social de la Escuela Graduada de Trabajo Social Beatriz Lasalle, y Doctorado de la Universidad Complutense de Madrid, Facultad de Filosofía, Programa de Filosofía, Ciencias de la Conducta y Sociedad. Tiene un Diplomado en Formación de Formadores en Educación Popular de la Universidad Antonio Ruiz de Montoya, Lima, Perú. Sus experiencias laborales más significativas son en Servicios Legales de Puerto Rico, Inc.; coordinación del Programa de Trabajo Social de la Universidad de Sagrado Corazón; y dirección del Departamento de Trabajo Social, Universidad de Puerto Rico, Humacao (UPRH). Es parte del personal docente de UPRH. Dedica su práctica privada al Trabajo Social Forense.

Recibido:

16 de mayo de 2014

Aprobado:

13 de septiembre de 2014 


\section{CONCEPTO HOSTOSIANO DEL SER HUMANO: ESBOZO PARA UN MODELO PUERTORRIQUEÑO DE TRABAJO SOCIAL}

"Tener antecesores gloriosos, tener ejemplares eternos de la humanidad virtuosa en sus anales, es feliz providencia de todos los pueblos que tienen historia; pero ser siempre merecedores de ellos y contribuir con su vida a hacerlos recordar perpetuamente, no es gloria que saben recoger todos los pueblos".

Eugenio María de Hostos

\section{Introducción}

El desarrollo del Trabajo Social en Puerto Rico y su sanción a nivel de la sociedad presenta la responsabilidad de continuamente mirar sus fundamentos y su práctica. Al decir Trabajo Social en Puerto Rico, necesariamente no quiere decir 
que sus fundamentos y su práctica tienen su raíz en lo que es la dinámica de ese contexto, ya sea cultural, espiritual, social, histórica, filosófica o geo-ambiental. Sin embargo, se parte de la premisa de que el Trabajo Social en su práctica tiene como punto de partida la sociedad y la cultura donde se ejerce.

El presente trabajo es un esbozo inicial de lo que viene siendo una preocupación continua en nuestro pensamiento: el Trabajo Social puertorriqueño. Nos preguntamos si se incorpora en su conceptualización a los pensadores que históricamente han interpretado esta sociedad, o que desde la experiencia han elaborado fundamentos sociológicos o de pensamiento universal a partir de nuestra realidad. No se trata de evadir a los clásicos, modernos o postmodernos que son utilizados y que en su mayoría son europeos o norteamericanos, con algunas inclusiones latinoamericanas. Es cuestión de incluir entre ellos a nuestros pensadores, sociólogos y filósofos y considerar el espacio que ocupan en el pensar del Trabajo Social en Puerto Rico. Muchos de los que han elaborado fundamentos filosóficos o sociológicos partiendo de la experiencia puertorriqueña responden a corrientes de pensamiento europeos o norteamericanos como Fray Iñigo Abbad y Lasierra con su Historia Geográfica, Civil y Natural de la isla de San Juan Bautista de Puerto Rico (S XIII), Oscar Lewis en su obra La Vida, analizando la pobreza en Puerto Rico, así como la Historia de Puerto Rico por Paul G. Miller (Chicago: Rand McNally). Sin embargo, tenemos aquellos pensadores que han hecho planteamientos que responden a las necesidades de los contextos sociales puertorriqueño, caribeño o latinoamericano. Podríamos mencionar entre los pensadores puertorriqueños algunos contemporáneos como Manuel Maldonado Dennis, conocido como historiador y ensayista, que en su obra sobre Eugenio María de Hostos, América: Lucha por la libertad (San 
Juan: Ediciones Compromisos, 1968), nos muestra un estudio sobre la obra de Eugenio María de Hostos como educador, libertario, antillano y latinoamericano. Entre sus obras más reconocidas están Puerto Rico Mito y Realidad (Barcelona: Península, 1968) y Un análisis socio-histórico de la emigración puertorriqueña (México: Siglo XXI, 1976). Igualmente José Emilio González, otro hostosiano, presenta la complejidad del Puerto Rico colonial en sus obras: El país de cuatro pisos y otros ensayos (Río Piedras: Ediciones Huracán, 1989) y Nueva visita al cuarto piso (Madrid: Libros del Flamboyán, 1987).

Luis Nieves Falcón se destacó como educador, sociólogo, político y luchador por la libertad y la justicia social, desde una perspectiva anticolonial y revolucionaria. Pensó la transformación de la realidad social puertorriqueña desde la educación, economía y la política. Fue maestro de maestros, forjador de luchadores por una sociedad justa, equitativa y libre de opresiones coloniales. Entre sus obras trabaja la realidad social puertorriqueña desde sus diferentes ángulos de especialidad: Un siglo de represión política en Puerto Rico 1898-1998 (San Juan: Ediciones Puerto, 2009), Diagnóstico de Puerto Rico (San Juan: Editorial Edil, 1972) y La luz desde la ventana (San Juan: Ediciones Puerto, 2002).

Juan Antonio Corretjer fue promotor del cambio social de forma revolucionaria/transformadora, parte de cuya obra ha sido recopilada en Juan Antonio Corretjer en CD (15 libros en PDF) (Ciales: Casa Corretjer). A Julia de Burgos se le conoce como poetisa que pensó desde sus versos las realidades humanas, sociales, de la pobreza y opresión puertorriqueñas, así como desde la realidad opresiva de la mujer caribeña. Sus expresiones no se circunscribieron a la poesía. Utilizó la epístola, el discurso y el periodismo. Encontramos algunas de 
sus cartas en la Revista A Propósito (Núm. 5, Año5 de marzo de 1996, Ponce, Puerto Rico. La mujer ante el dolor de la patria fue un discurso suyo publicado en la Revista del Ateneo Puertorriqueño (1995).

De forma más directa en nuestros análisis es indispensable contar con el legado de las pensadoras que ha producido la propia profesión, como lo son Carmen Rivera Alvarado, pionera del Trabajo Social en Puerto Rico, y la trabajadora social y profesora Raquel Seda, con sus análisis sobre la profesión y las políticas sociales. Es de mencionar también José Joaquín Parrilla, quien miró la realidad de Puerto Rico desde una perspectiva contestataria, nutriendo al Trabajo Social con un pensamiento obrerista como se ve en su biografía.

La realidad puertorriqueña, según estos pensadores, es vista desde miradas críticas que llevan a propuestas de lucha, de transformación social, política y económica. Algunos han presentado propuestas liberadoras y anticolonialistas que aún sirven de marco a luchas populares. Han sido pensadores que han trascendido sus análisis de la realidad puertorriqueña y han demostrado su compromiso a través de insertarse de formas diversas en luchas sociales y políticas.

Son muchos los(as) pensadores que con mayor o menor visibilidad han presentado análisis y visiones de la realidad social puertorriqueña que reta las versiones oficialistas. Uno de esos pensadores históricos es Eugenio María de Hostos con su visión cósmica del ser humano, la abolición de la esclavitud, y particularmente el respeto por la educación de las mujeres. Hostos ha pensado la realidad social puertorriqueña de forma tal que incide históricamente en un pensamiento de lucha propio del Trabajo Social. 
Estaremos rescatando una mirada de algunos conceptos del pensamiento hostosiano que contribuirían a una clarificación de la práctica del Trabajo Social desde la realidad puertorriqueña.

Cuando hablamos de la elaboración de un modelo a partir de uno o unos conceptos particulares, no pretendemos hacer un calco exacto del pensamiento que pretendemos incorporar. Al acercarnos al pensamiento de un sociólogo, filósofo o trabajador social, es obligado conocer, antes que nada, la esencia de su pensamiento, ubicarlo históricamente y luego incorporarlo en sus elementos fundamentales.

Mucho nos falta, como profesionales del Trabajo Social en Puerto Rico, por entendernos a nosotros mismos y los caminos de nuestros pensadores. Conocernos y entender cómo se nos ha pensado es una tarea importante para el Trabajo Social, así como socializar a través de la investigación y el diálogo las formas como conceptualizamos las relaciones sociales en el Puerto Rico de hoy y de cada instancia. El pensamiento del cual parten los conceptos y herramientas desde las cuales conformamos la profesión, van a ser determinantes en qué tipo de Trabajo Social estamos practicando y cuáles son los fines sociales a los cuales corresponde. No es lo mismo construir la solidaridad desde la hospitalidad cultural puertorriqueña a hacerlo desde un sentido de inferioridad colonial. Eso nos lleva al reto, como profesión, de profundizar el conocimiento construido desde nuestra realidad social puertorriqueña. Es nuestro, también, el deber de mantener el análisis continuo de la sociedad puertorriqueña, desde lo que somos y de lo que queremos ser, y de cuáles fines y medios estén supeditados al bien común. 


\section{El concepto hostosiano del ser humano}

Eugenio María de Hostos parte del individuo como centro y totalidad junto a la naturaleza de sus relaciones sociales en diversos contextos. Él entiende que el deber del ser humano es el bien común, al que se refiere como bien social. El bien social es aquel en el que el beneficio individual se ve en virtud del beneficio social. Esa es una ley que él incorpora a la naturaleza social del ser humano, que se rompe cuando se mediatiza con relaciones sociales denigrantes y esclavizadoras. Es una ley que bien pudiera ser uno de los principios del Trabajo Social puertorriqueño. Ciertamente podría ser una guía interna para el principio de justicia social.

Eugenio María de Hostos plantea una visión del ser humano que llama "visión cósmica". Esa visión integral establece que el ser humano y la naturaleza son uno. El cuerpo cósmico pone al ser humano y a la naturaleza en un mismo plano. Indica que para conocer la sociedad, tenemos que necesariamente conocer la relación entre la sociedad y la naturaleza. Hostos explica la relación de los fenómenos sociales entre sí de forma tal, que internamente la sociedad está compuesta de unos elementos de contexto en los que se da la conducta humana y se establecen las relaciones humanas íntimamente ligadas a su relación con los fenómenos de la naturaleza. La Ley de la Sociabilidad establece que no importa el contexto en que se den las relaciones sociales, estas responden a la naturaleza gregaria del ser humano. A partir del individuo, lo natural es que establezcamos relaciones sociales en familias, grupos, comunidades, organizaciones, regiones, comarcas y globales. En el caso de Puerto Rico, él específicamente habla de que lo natural para nosotros es ser parte de las Antillas y de la comunidad de naciones iberoamericanas. 
Hostos propone, al decir de su maestro Krause, que el ideal político era la humanidad concebida como una federación de asociaciones universales en las que no se sacrificarían las peculiaridades de sus componentes. Estuvo en su tiempo muy consciente de las opresiones y desigualdades de los pueblos y la organización política u ordenamiento social que proponía y atendía las injusticias propias de la época como el colonialismo, la esclavitud, la pobreza y la libertad.

El segundo factor social que Hostos incluye como factor de orden es el trabajo. El trabajo cósmico lo iguala por un lado con los procesos continuos de cambio a nivel de astros del Universo. El trabajo humano lo categoriza como que puede ser espontáneo y reflexivo. En términos cósmicos es el trabajo, tanto en naturaleza como en la sociedad, lo que impulsa el cambio. Hostos indica, además, que los cambios son diferentes en su naturaleza. Los hay imperceptibles, invisibles, latentes, así como en su dimensión pueden ser mínimos y máximos, que en términos del universo son constantes. En lo relacionado al ser humano, que es una continuación del cosmos, es producto del trabajo. Estas fuerzas invisibles no están reconocidas, y en la mayoría de las ocasiones no están en la conciencia generalizada del pueblo. En la sociedad contemporánea se puede hablar de las fuerzas del neoliberalismo y de la globalización, que tiene como características la deslocalización de su funcionamiento, los polos múltiples del poder y la fluidez del manejo del capital. Es un capital cuya nación es el dinero y su lealtad es hacia la acumulación del mismo. Muy bien dice Hostos que en los intercambios económicos, no solo se intercambian bienes materiales, si no también hay una transmisión de valores.

En una sociedad en la que se plantea el fin del trabajo, Hostos preguntaría quienes de forma invisible o visible están constantemente laborando para que el trabajo en la sociedad 
tome formas esclavizadoras. Así como el trabajo produce cambio de forma ascendente para el fin de bien social, puede también obrar en una forma humanamente descendente, fomentando las relaciones opresivas en los diferentes contextos.

Hostos (1989) llama a la libertad su segunda ley orgánica de la sociedad. La resume diciendo que:

"Esta Ley de Libertad actúa manifiestamente en el orden social, como actúa en el individual: en modo que en el ejercicio libre de nuestra actividad está una parte del bien a que aspiramos, y en la depresión de nuestra libertad está una parte del mal que es de nuestra naturaleza combatir" (P. 151).

La lucha por la libertad está vinculada a la posibilidad del ser humano de ejercer su "libre albedrío" y romper con relaciones de esclavos, y está relacionada con el respeto por la dignidad el ser humano. Él ve la lucha por la libertad como parte consustancial del ser humano y no hacerlo es ir contra ella y descender a niveles inferiores de nuestra humanidad. Se traduce el trabajo social como la posibilidad del ser humano de ejercer su autodeterminación.

Al colocar en el mismo plano las dimensiones bio-sicosociales-culturales-espiritual-geo ambiental debemos preguntarnos: ¿Cúal es el contexto? ¿De qué hablamos cuando en Trabajo Social establecemos la importancia del contexto? De acuerdo a esta visión cósmica, el contexto sería lo relacional. Hostos habla de contexto cuando hablamos de cuáldónde-cómo se dan las relaciones: personas, familias, grupos, comunidades, organizaciones, sociedad-regional-internacional. En esos contextos, los seres humanos interactuamos y los configuramos desde lo que constituye nuestra humanidad 
compuesta por las dimensiones en un todo. Hostos (V. III) indicaría "que toda actividad de las sociedades humanas consta en un hecho general o genérico, que es la civilización" (p.136). Indica también que toda la eficacia de las leyes naturales de la sociedad estriba en la fuerza con que actúan las funciones de la vida social, que no son ninguna otra cosa que manifestaciones de ley de vida.

Es interesante que Hostos (V. VIII) también nos indique "que los órganos que componen el ser social son: el individuo, la familia, el municipio, la provincia, comarca o región, sociedad nacional y sexto, la humanidad" (p.88). En su concepción del ser social se aleja de un análisis lineal cuando reconoce que las operaciones y funciones del individuo distan mucho de las funciones de la sociedad, y que la sociedad está muy lejos de ser la suma de todos los individuos, dándole vida propia a lo social. Él indica que la base de la sociedad es el individuo, pero el medio es la asociación o relación establecida entre ellos. El medio para él es la familia, el municipio, etc. en las que se establecen unas relaciones particulares, unas asociaciones determinadas que establecen la mutua relación que requiere el ser humano como ser social y que también requiere la humanidad como un todo.

Hay formas de conceptuar el trabajo social que no incluyen las sociedades como un contexto; se queda en personafamilia-grupo-comunidades-organizaciones. Si tomamos como ejemplo algunos textos utilizados para la formación de trabajadores sociales, se puede observar el ámbito que según los autores cubre este contexto. Un libro de texto utilizado es el de Charles Zastrow y Karen K.(2007) Kirst-Ashman. Estos abordan la conducta humana desde una perspectiva de ciclo de vida, conceptualizando en un nivel macro a las comunidades y las organizaciones, en el nivel mezzo las familias y grupos pequeños, y en el micro la persona. El cambio social no es 
considerado dentro de su esquema sistémico. Ese mismo esquema se encuentra en Dean Hepworth, Ronald Rooney, Glenda Dewberry Rooney, Kimberly Strom-Gottfried y JoAnn Larsen (2006).

Sin embargo, Ralph Anderson \& Irl Carter (1994) presentan un esquema diferente en el que, además de los otros contextos mencionados, presentan la cultura como un fenómeno grupal, así como la sociedad. Una vez se integran la cultura, la sociedad y lo global como contextos en los cuales se dan las relaciones humanas, se pueden considerar como lugares posibles para la transformación, de acuerdo a los principios de la profesión y necesidades que producen las formas en que están construidas u organizadas dichas relaciones. Dejarla fuera implica que no se considera el cambio de la sociedad ni la crítica de la cultura. Para el trabajo social debiera ser problemática esa exclusión porque son contextos en los que se dan muchas de las opresiones y relaciones esclavizadoras que se proyectan sobre los demás contextos, ya que son mutuamente dependientes dentro de la concepción integral del ser humano. Los autores mencionados que tienen un conceptual que deja fuera la sociedad, la cultura y lo global tienden a proponer un enfoque más de mantenimiento que de cambio.

La globalización se discute aparte de lo que son los contextos para la conducta humana. Las sociedades no se incluyen como un contexto en el cual considerar la conducta humana. Particularmente en la formación sobre conducta humana se observa que los niveles sociedad-regional-global no forman parte de esos contextos. Ciertamente, en sociedades en las que se parte del mantenimiento y balance de la sociedad, en la que los cambios que se promueven son para el mantenimiento de un funcionamiento socialmente aceptado, 
no se incluye el cambio en las relaciones a nivel de sociedad ni a nivel global. Tenemos que considerar que lo socialmente aceptado en sociedades como la nuestra no siempre está dirigido al bien común, si no a que la conducta sea un medio para los fines del capital o de los sectores dominantes, que por lo general vienen siendo lo mismo.

Hostos giró su foco más allá de lo inmediato, de lo localnacional; tuvo una mirada que Ulrich Beck (2009) llamaría glocal. Aunque en su momento no se discutía la globalización como relaciones sociales en el contexto, él sí entendió, en su práctica, la necesidad de vernos como parte de las Antillas y más aún como latinoamericanos, asomándose así en él lo global como solidaridad e identidad. El bien común y la vida social debería tomar en consideración la realidad de que no estamos solos en el mundo y que hay unas realidades sociales que son hermanas y deben ser consideradas como parte del radio de acción propio. En su pensamiento incluyó no solo la conceptualización de lo que somos, si no también propuso que se trabajara hacia darle concreción a esa realidad social natural, a través de una Confederación Antillana.

En su "Carta al presidente del Perú", que fuera publicada en El Argentino de Buenos Aires el 13 de octubre de 1873 (Manuel Maldonado Dennis, 1976), Hostos decía que:

“...las Antillas desempeñan en el plan natural de la Geografía de la civilización el papel de intermediarias del comercio y de la industria: el comercio es actividad aplicada a las necesidades, la industria es ciencia aplicada al bienestar de los hombres, y son conductores de ideas, como lo son de elementos físicos y de bienestar; trasmisores de progresos morales e intelectuales, como lo son de progresos materiales" (p.17). 
Aún en su mirada de las Antillas, actuando como conjunto, refleja esa visión integral en la que lo material se constituye en un medio para el bienestar del ser humano, no un fin en sí mismo.

Además de mirar la sociedad puertorriqueña, existen otras visiones del ser humano. Está la visión de pueblos indígenas en países latinoamericanos. Estas grandes poblaciones indígenas incorporan al concepto del "Buen Vivir" con la visión del ser humano supeditado a la naturaleza. Estos pueblos en su expresión de lo que debe ser una vida balanceada ven al ser humano sometido a la "Pachamama", "Madre Tierra" como la gran madre que da vida y protege a sus hijos. Esa visión ha sido incorporada como visión de mundo y del ser humano en la Constitución Ecuatoriana. Nos dice Patricio Raza de El Ecuador (2010):

"...el buen vivir, el cual se construye desde la búsqueda de la igualdad y justicia social, desde el reconocimiento, la valoración y el diálogo de los pueblos y de sus culturas, saberes y de vida.....en intima armonía y relación con el planeta y el universo" (p.59).

Hay diversas visiones del ser humano desde las cuales se construyen acciones y relaciones sociales particulares. Esas visiones pueden marcar los fines y los medios del accionar de una sociedad.

Con la visión hostosiana del ser humano podemos relacionar en cierta manera la perspectiva con dimensiones bio-sico-socialcultural-espiritual-geo-ambiental. Esta perspectiva aborda la conducta humana producida por un ser humano integral. Desde esta perspectiva, la conducta es influenciada por sus diversas dimensiones en interacción y mutua dependencia, 
colocadas estas en igualdad de condiciones. Esa visión integral del ser humano y la conducta humana influenciada por las diversas dimensiones del ser humano reconocen que en determinadas conductas predomina la influencia de alguna de las dimensiones sobre otras. En ocasiones vemos lo biológico influenciando sobre la conducta de un ser humano y reflejarse en las dimensiones sicológicas, sociales, espirituales, etc. También vemos cómo el daño a la naturaleza y el uso de esta como medio para el capital afecta las dimensiones biológicas, sicológicas y espirituales, y las relaciones sociales del ser humano. En esa realidad descansa la singularidad del ser humano y su conducta.

Como profesión y como sociedad, en ocasiones por momentos históricos o prioridades y valores sociales, le restamos importancia a alguna de esas dimensiones. En la sociedad neoliberal en la que se pretende que las fuerzas del mercado rigen la vida del ser humano en sus diversas dimensiones, no se les presta importancia a alguna de las dimensiones como es la espiritual, la geo-ambiental. El individualismo, el consumismo van por encima como valores. No se considera en nuestra ponderación social el impacto de la organización social, el distanciamiento de los seres humanos en virtud de aumentar la producción para aumentar el capital y la destrucción de la naturaleza en función del capital sin importar que la destrucción de aire, tierra, aguas disminuya las posibilidades de que el ser humano como ser integral pueda sobrevivir.

Se vive en una sociedad de medios, en la que el ser humano está desintegrado y su concepción cósmica queda, en sus dimensiones, supeditada a los fines del capital. Tal parecería que el ser humano, como manipulador de la naturaleza, se ha puesto por encima de ella, pero realmente le ha dado la 
espalda y la apariencia de que sus descubrimientos científicos y tecnológicos lo han llevado a dominarla es solo una ilusión. Realmente la naturaleza está siendo utilizada como instrumento para la consecución de los fines del capital y no para el bien común. Si la naturaleza y sus leyes fueran tratadas como una dimensión más de la visión integral de todos los seres humanos y no como elementos a los que se les puede hacer daño, no tendríamos de vuelta daño de parte de ella.

\section{Modelo para la práctica del Trabajo Social}

\section{Fundamentos para la práctica del Trabajo Social y su concepto del ser humano}

En este modelo se parte de la visión integral del ser humano o al decir de Hostos, una "visión cósmica". Se entiende el ser humano en un todo con la naturaleza en interacción con los diversos contextos sociales en los que se producen las relaciones y conductas sociales. Se considera al ser humano como gregario por naturaleza y sus relaciones sociales abarcando los contextos que constituyen la humanidad, con el ser humano en el centro. Los contextos en que se dan las conductas humanas son las familias, grupos, comunidad, organizaciones, sociedad, lo global.

\section{Contextos de la conducta humana en su naturaleza dinámica}

Para todos los contextos en los que se construye la conducta humana, hay unos elementos que la componen y que inciden en ella. No son diferentes para cada contexto: familia, grupo, comunidad, organizaciones, sociedad, global. 
La conducta y las relaciones sociales tienen manifestaciones distintas: la historia familiar y la historia organizacional tienen el elemento temporal con sus propios componentes y su impacto particular en el contexto del momento en que se trabaja la conducta y las relaciones sociales. Así también, como parte de esos contextos. se considera el concepto del ser humano del que se parte, su historia, la diversidad humana, elementos de las relaciones económicas y políticas. En el todo, estos elementos interactúan entre sí, y unos tienen mayor preponderancia y pertinencia que otros.

Este contexto tiene unas manifestaciones que pueden considerarse como productos o construcciones de las relaciones sociales en cada contexto. Así hablamos de las relaciones humanas que se constituyen en cultura organizacional, cultural nacional o comunitaria, de una región o pueblo. Igualmente se habla de relaciones de poder desde la familia, las organizaciones, sociedades, lo global, etc. En este modelo no se utilizan los métodos como punto de partida para la intervención, ni los sistemas humanos, como se diría, bajo un concepto sistémico. Se consideran contextos para las relaciones sociales y esas relaciones se construyen de formas diferentes de acuerdo a los fines y medios. Los contextos se distinguen por sus identidades. En el contexto de la sociedad puertorriqueña con el cuestionamiento, tergiversación de nuestra historia e identidad cultural, con la xenofobia, machismo, la violencia de género, racismo, homofobia, la construcción de las identidades a todos los niveles son importantes. Estas construcciones en nuestra sociedad reflejan luchas de poder, elementos de poblaciones vulnerables y marginaciones por lo que deben tener visibilidad en los análisis para la práctica del Trabajo Social. 


\section{La relación profesional}

Desde el inicio del establecimiento de la relación profesional, entran en juego varios elementos: el uso de la persona, la intención de la trabajadora o trabajador social de utilizar su intervención para un proceso de cambio o transformación. Desde sus inicios, el camino a seguir se hará respetando la autodeterminación de la persona o personas con quien se trabajará. Desde este punto se inicia el proceso de transformación y se ponen en funciones las competencias requeridas por el trabajo social.

Los hilos conductores de este proceso son el cambio social en cuanto a contenido y método, y los principios de la profesión que problematizan de los contextos y las relaciones sociales para el establecimiento de los fines y medios para la intervención.

Esto lleva a la reflexión y evaluación inicial como base para la intervención. Se emite juicio profesional junto a las personas involucradas de acuerdo al contexto en que se dan las relaciones sociales y se procede a la planificación participativa.

\section{Acción o intervención profesional}

Es el ámbito para la selección de bases teóricas relacionadas a la situación o situaciones atendidas, así como los modelos pertinentes. Se entiende como intervención a la etapa en que se llevan a cabo las acciones profesionales que junto a las personas con las que se trabaja, son promotores del cambio. Es el ámbito en que se elige la teoría de la práctica, siendo el momento de la acción transformadora el tiempo-espacio en que se avalúa la eficiencia y la efectividad, con todos los elementos que implique. En el espacio más identificado con el trabajo social, por lo general se considera la profesión como una de acción, de métodos, y no una teórica y creadora de conocimientos. 


\section{Reflexión continua}

Implica el cuestionamiento del conocimiento práctico adquirido sobre la realidad, una vez inmerso en ella. Se reflexiona sobre contenido y proceso. Se realizan correcciones a partir de los conocimientos iniciales, reflexiones continuas y los fines-acciones futuros. Demás está decir que la solidez teórica, metodológica y de principios del profesional es lo que permitirá una reflexión pertinente, además de su capacidad para construir un proceso participativo que permita a las personas con quienes se trabaja que puedan nutrir el proceso, para así darle pertinencia a la intervención.

\section{Terminación y seguimiento}

Puede ser el momento de cierre de la intervención o un momento de evaluación para continuar el proceso a otro nivel. La terminación se debe abordar con tiempo, considerando que el fin de la intervención social particular no será la última vez que esta(s) persona(s) trabajará(n) alguna necesidad y opresión en su(s) proceso(s) de vida y consecución de la justicia social.

Este modelo debe considerarse una guía para una intervención con intención de cambio. Lo importante es lo que se quiere lograr. Debe verse con flexibilidad y no necesariamente secuencial. Ciertamente, hay unos elementos que vienen primero que otros, pero una vez se inicia el contacto con los contextos en los que se va a trabajar, la planificación de la intervención no está prescrita. Se acuerda entre los involucrados con la guía del/la profesional.

Este es un modelo para guiar a profesionales del trabajo social. Se observa que no incluye el trabajo multidisciplinario, que es característico de la práctica profesional de trabajadores sociales. La relación con otros profesionales debe considerar la compatibilidad de los modelos que utilice 
cada profesional. Es tarea del trabajador y trabajadora social utilizar la flexibilidad el diálogo para insertar los principios de la profesión como factores que problematizan en el proceso de la colaboración multidisciplinaria. Los factores formación profesional e identidad profesional fortalecida permitirán que en el trabajo multidisciplinario, el trabajo social tenga el espacio correspondiente y no subordinado.

Un modelo es siempre una guía. No debe ser una camisa de fuerza para el/la profesional guiado(a) por los principios del trabajo social. La formación sólida del trabajador(a) social le permitirá emitir un juicio profesional adecuado a las relaciones sociales con las que trabajará, y le permitirá adecuar el proceso a las necesidades e intereses pertinentes.

En nuestra formación continua debemos pensar críticamente nuestras acciones y anclarlas cada vez en lo que constituye la sociedad puertorriqueña, nuestra historia, nuestros valores. El hacer nuestra práctica cada vez más cercana a lo que somos contribuye a la pertinencia de nuestra profesión. 


\section{Referencias}

Anderson, R. \& Carter, I. (1994). La Conducta humana en el Medio Social: Enfoque sistémico de la sociedad. Gedisa: Barcelona.

Arpini, Adriana. (2007). Eugenio María de Hostos y su época: categorías sociales y fundamentación filosófica. Editorial Universidad de Puerto Rico. Río Piedras.

Beck, Ulrich. (2009). La sociedad del riesgo global. Madrid: Siglo XXI

Carrión Maldonado, lleana. (2014) Sistematización de la Experiencia de Supervisor-Supervisado en la Instrucción Práctica de Estudiantes de Trabajo Social en Adiestramiento 2013-2014, en el Proyecto PRODECO, Bo. Jagual de Gurabo. Investigación inédita.

Código de Ética Profesional (2010). Colegio de Profesionales del Trabajo Social de Puerto Rico. De Hostos, Eugenio María. Obras Completas Edición Crítica: Eugenio María de Hostos. Editorial Universidad de Puerto Rico: Río Piedras. Tomo I, Vol. VIII, IX.

Hepworth, D. \& otros. (2006). Direct Social Work Practice: Theory and Skills. Thomson: USA.

La Educación Popular en la lucha por el buen vivir. (2010). Asamblea Intermedia de Bartolina Sisa Mélida Amaya Montes y Monseñor Romero. CEAAL: El Salvador

Maldonado Dennis, Manuel. (1988). Hostos, Eugenio María de. América: Lucha Por La Libertad. Editorial Compromiso: San Juan.

Robbins, S.\& Chatterjee, P.\& Canda, E. (2006). Contemporary Human Behavior Theory: A Critical Perspective. Pearson: New York.

Zastrow, C. \& Kirst-Ashman, K. (2007). Understanding Human Behavior and the Social Environment. Thomson: USA. 